\title{
PARÂMETROS BIOQUÍMICOS, HEMATOLÓGICOS E IMUNOLÓGICOS INDICARDORES DE ESTRESSE EM ESTUDANTES UNIVERSITÁRIOS
}

\author{
BIOCHEMICAL, HEMATOLOGICAL AND IMMUNOLOGICAL PARAMETERS STRESS \\ INDICATORS IN UNIVERSITY STUDENTS
}

\author{
Annie Silva Lima', Bianca de Brito Costa ${ }^{1}$, Francielle Bonet Ferraz ${ }^{2}$ \\ ${ }^{1}$ Bacharel em Biomedicina; ${ }^{2}$ Professora doutora em Biociências e Biotecnologia. \\ Instituição: ' Universidade do Estado do Pará (UEPA); ${ }^{2}$ Universidade do Estado do Pará (UEPA) e \\ Faculdade Carajás.
}

Autor correspondente: Ferraz, Francielle Bonet - francielle.bonet@gmail.com - Telefone: 5594 992763125 - E-mail: francielle.bonet@gmail.com

UEPA - Avenida Hiléia, s/no, Agrópolis do Incra, Bairro Amapá, Marabá - Pará - Brasil. CEP: 68502-100.

\section{RESUMO}

O estresse é uma condição muito comum na sociedade, principalmente em estudantes do ensino superior onde se assola muitas dificuldades durante o curso, e ainda, ocorrendo constantemente pode acarretar várias comorbidades, não somente em estudantes, mas em vários indivíduos que continuamente sofrem por situações de estresse em seus cotidianos. Assim, dividiu-se o estresse em duas etapas: a Síndrome de Adaptação Geral, que pode ser explicada como um conjunto de respostas imprecisas de defesa e de Adaptação Orgânica ao Causador, onde é dividida em três partes, que são as fases do estresse, Fase de Alarme ou Alerta, Resistência e Exaustão. Este trabalho tem o quesito principal de investigar a incidência e a influência do grau de estresse nas possíveis alterações bioquímicas, hematológicas e imunológicas em alunos universitários por meio de revisão bibliográfica, buscando então por meios de artigos científicos disponibilizados em diversas bases de dados. A observação dos estudos selecionados foi realizada de forma descritiva, facilitando analisar, computar, descrever e classificar os dados, com a intenção de reunir o conhecimento produzido sobre o tema e estimular um panorama holístico sobre como as situações de estresse são capazes de possibilitarem alterações bioquímicas e hematológicas, tornando mais propícia a diminuição da qualidade de vida de um indivíduo Palavras-chave: Estresse psicológico; sistema imunológico; estresse fisiológico.

\begin{abstract}
Stress is a very common condition in society, especially in higher education students, who experience many difficulties during the course, and even if it occurs constantly, it can cause several comorbidities, not only in students, but in several individuals who continually suffer from stress in their daily lives. Thus, stress was divided into two stages: the General Adaptation Syndrome (SAG), which can be explained as a set of inaccurate defense responses and Organic Adaptation to the Cause, where it is divided into three parts, which are the phases stress, Alarm or Alert Phase, Resistance and Exhaustion. This work has the main question of investigating the incidence and influence of the degree of stress on possible biochemical, hematological and immunological changes in university students through bibliographic review, then searching for scientific articles available in several databases. The observation of the selected studies was carried out in a descriptive manner, facilitating the analysis, computation, description and classification of data, with the intention of gathering the knowledge produced on the subject and stimulating a holistic panorama on how stressful situations are capable of enabling biochemical changes. and hematological, making it more conducive to a decrease in the quality of life of an individual.

Keywords: Psychological distress; immune system; physiological stress.
\end{abstract}




\section{INTRODUÇÃO}

O estresse, é caracterizado como um mal comum na sociedade do século XXI, sendo marcado por vários aspectos de ansiedade, depressão, sendo físico (dor, choque, exposição ao frio), químico (por ação de drogas) ou psicológico (distúrbios sociais e situações de medo) (PAGLIARONE; SFORCIN, 2009).

O estresse psicológico pode ser evidenciado por alguns marcadores como cortisol, glicose e também por células sanguíneas uma vez que durante os processos de estresse fica evidente alterações nas concentrações séricas de cortisol e glicose, tornando-os marcadores bioquímicos e hematológicos de estresse (FERRAZ; GOMES, 2009; LEITE et al., 2016; STURION, 2011).

$O$ estresse pode ser dividido em duas etapas: a Síndrome de Adaptação Geral (SAG), que pode ser explicada como um conjunto de respostas imprecisas de defesa e, de Adaptação Orgânica ao Causador ( $A O C$ ), a qual é dividida em três fases, que são as fases do estresse, determinada por Selye (1936). A primeira é denominada fase de alarme ou alerta, que caracteriza o momento em que o organismo humano reconhece o agente causador de lesão e prepara uma resposta orgânica rápida para o enfrentamento de luta e fuga, entretanto, permanecendo por pouco tempo, para manter o sistema em harmonia. Após esta etapa, passa-se para a segunda fase chamada de fase de resistência: o organismo tenta uma adaptação, buscando a homeostase, caso o organismo consiga resistir e possua estratégias para eliminar o agente estressor, o organismo consegue cessar o estresse entretanto, se o estressor é contínuo e o organismo não possuir estratégias para enfrentá-lo, o indivíduo está mais exposto a doenças seguida de instabilidade emocional (PRADO, 2016).

Na terceira e última fase, a fase de exaustão, cujo agente causador permanece no organismo pois não foi possível eliminá-lo ou adaptar-se a ele, é considerada a fase mais crítica. Devido a várias exposições ao agente estressor a fase de alarme fica mais evidente, ou seja ela fica presente de uma maneira mais acentuada podendo acarretar em um colapso, tornando assim esse organismo mais suscetível a várias doenças que envolvem o estresse, algumas leves e outras mais graves como psoríase, úlceras, alterações de sono e vigília, transtorno de ansiedade e depressão (PRADO, 2016; LIPP; MALAGRIS, 2011).

Frente a estas respostas fisiológicas complexas, o presente trabalho tem por objetivo explanar de forma holística a influência do estresse nos parâmetros bioquímicos, hematológicos e imunológicos e suas prováveis implicações no processo saúde/doença em alunos de ensino superior.

\section{METODOLOGIA}

Trata-se de uma revisão sistemática sobre o estresse e sua interferência fisiológica, particularmente relacionada aos alunos de graduação no ensino superior. Para o levantamento dos artigos na literatura, realizou-se uma busca nas bases de dados Literatura Latino-Americana e do Caribe em Ciências da Saúde (LILACS) e Medical Literature Analysis and Retrieval Sistem on-line (Medline), Scientific Electronic Library Online (SciElo) e Google Acadêmico.

Partindo disso, as narrativas utilizadas para a pesquisa, foram "estresse", "parâmetros imunológicos", "psiconeuroimunologia", "cortisol", "marcadores de estresse", "marcadores de ansiedade", "estresse psicológico" buscando-as nas línguas portuguesa e inglesa.

As especificações de inclusão definidas para a seleção dos artigos são artigos publicados em português e inglês, artigos na íntegra que retrata a temática referente à revisão sistemática e artigos publicados e indexados nos referidos bancos de dados nos últimos onze anos, podendo haver exceções com datas posteriores.

A análise dos estudos selecionados foi realizada de forma descritiva, possibilitando observar, contar, descrever e classificar os dados, com o intuito de reunir o conhecimento produzido sobre o tema explorado na revisão.

\section{DO ESTRESSE ÀS ALTERAÇÕES FISIOLÓGICAS}

A priori, Hans Selye, no século $X X$, criou o termo estresse, considerando que este pode ser definido como um estado manifestado por uma síndrome específica, seja ela física, psicológica ou comportamental, onde cada indivíduo, com suas peculiaridades cotidianas, manifestará um conjunto de sintomatologias inerentes ao seu dia a dia (HIRSCH et al., 2018). Junto a isso, pouco depois, criou as três fases do estresse (Alarme, Resistência e Exaustão), muito estudada atualmente por Lipp.

Com Lipp (2011), foi-se discutido sobre mais uma fase além das três já existentes (Alarme, Resistência e Exaustão), a fase de quase-exaustão, 
onde pode-se observá-la entre as duas últimas fases, dito como um estado anormal de fraqueza, o indivíduo se torna inapto a gerar reações e resistir a estressores. Colaborando assim com o aparecimento de algumas sintomatologias leves como tremor muscular, desânimo, falta de memória e atenção e as mais graves por exemplo o aumento da frequência cardíaca e respiratória, todavia este corpo pode então entrar em colapso e assim levar a óbito (PRADO, 2016).

Sabe-se hoje que o estresse está estreitamente ligado com o cortisol, um glicocorticoide que é produzido pelas glândulas suprarrenais, podendo interferir em sistemas, como por exemplo o imunitário, onde este gera um efeito negativo nas suas células (FERRAZ; GOMES, 2009). Esse ciclo inicia no eixo essencial que gera uma cascata, passando pelas fases do estresse, até o cortisol. O eixo hipotalâmico-hipofisário-adrenal (HHA), que é composto pelo hipotálamo e hipófise, localizados na parte inferior do tálamo no córtex cerebral, e pela glândula adrenal, que se localiza na parte superior dos rins. Estes secretam vários hormônios muito ligados ao sistema imunológico, De outra perspectiva, essa modulação do eixo HHA também pode ocorrer pela ligação aos receptores de mineralocorticoides (RM) e glicocorticoides (RG) nos tecidos do eixo, com isso ficará exacerbada toda a demanda de glicocorticoides (JURUENA; CLEARE; PARIANTE, 2004).

O eixo HHA começa liberando os hormônios: hormônio liberador de corticotrofina (HLC) e arginina vasopressina (AVP) do hipotálamo, que juntos conseguem ativar a liberação da secreção do hormônio adrenocorticotrófico (ACTH) da glândula hipófise, que consegue promover a secreção de glicocorticoides pelo córtex da adrenal, com isso os glicocorticoides conseguem se conectar a seus respectivos receptores e ordenar sua funcionalidade sobre os tecidos, e assim se comunicar com o HHA onde são então responsáveis pela inibição negativa por feedback da secreção do ACTH pela hipófise e do HLC a partir do hipotálamo (JURUENA; CLEARE; PARIANTE, 2004).

Outros hormônios como a serotonina, que fornece uma estimulação no HLC, por meio dos subtipos de receptores de 5-hidroxitriptamina (5HT1A, 5-HT1B, 5-HT1C, 5-HT2). No entanto, a norepinefrina tem uma aplicação divergente, sendo estimulatória em doses mais baixas (via receptores a1) e inibitória em doses mais elevadas (via receptores $\beta$ ). A dopamina também é um neurotransmissor que interfere na ação dos "hormônios do estresse", onde realiza a supressão da liberação de aldosterona e noradrenalina por um mecanismo pré-sináptico inibidor nos gânglios simpáticos, com isso provoca a excreção de sódio e consequentemente, gera vasodilatação da artéria renal e mesentérica, resultando na regulação da liberação da acetilcolina (JURUENA; CLEARE; PARIANTE, 2004).

Entre as fases do estresse, hormônios diferentes são liberados. Onde a primeira se fixa com a produção de catecolaminas, adrenalina e noradrenalina, esses são exemplos de hormônios monoaminas, estas têm o poder de preparação dos tecidos como o miocárdio, para receber uma intensa atividade e resistir ao estresse (FONSECA; GONÇALVES; ARAÚJO, 2015). Isto pode afetar diretamente na fisiologia, fazendo com que a frequência cardíaca aumente, vasoconstrição, inibição da insulina e aumento da glicose sanguínea, por exemplo (GUEST et al., 2013).

Na segunda o HLC é liberado, e estimula a liberação ACTH, o qual este é levado até a adrenal, juntamente com a liberação de cortisol advindo do córtex da adrenal, o que eleva seu estado na circulação sanguínea e, esta elevação dos níveis de cortisol possibilita na inibição da liberação de ACTH e HLC em um modo de retroalimentação de rejeição (GUEST et al., 2013).

Na terceira fase os glicocorticoides já produzem um outro efeito, com o aumento da gliconeogênese e lipólise, por consequência, elevase os volumes de glicerol e ácidos graxos no sangue, além disso os glicocorticoides podem também estimular a mobilização e desaminação de proteínas do músculo esquelético. Com isso, o cortisol fica ainda mais elevado por muito mais tempo, gerando um estímulo a muitas comorbidades.

As células imunitárias também são bastante afetadas. Com os níveis de estresse elevado, tem-se uma migração exacerbada dessas células para os tecidos, fazendo com que essas quantidades na circulação sanguinea aumentem bruscamente. Fica evidente que algumas delas chegam primeiro ao sítio interesse, como o monócito que é responsável pelo reconhecimento de antígenos e atividade citotóxica fagocítica e como o neutrófilo, que são fagócitos de bactérias (FERRAZ; GOMES, 2009).

Os linfócitos também são liberados na circulação sanguínea, onde cumprem seu papel de 
identificação de infecções virais com o reconhecimento de $\mathrm{MHC}$ de classe I, fazendo também a identificação dos microrganismos e de agentes estressores. Os linfócitos podem sintetizar hormônios como ACTH e o GH, portanto o estresse em níveis elevados pode acarretar o aumento dos órgãos linfóides (FERRAZ; GOMES, 2009).

A glicose também tem seu ponto de ação no estresse. A regulação dos índices glicêmicos, é determinado por 3 fases interligadas: a produção de glicose no fígado; a captação dessa glicose e sua utilização por músculos, principalmente esqueléticos e tecidos periféricos; e as ações de hormônios, como insulina e hormônios contrarreguladores como glucagon, catecolaminas e cortisol (MAITRA; ABBAS, 2016). Sua influência sobre o estresse gera, a interrupção da homeostase glicêmica a partir da resposta fisiológica de "luta ou fuga" proporcionada pelo estresse devido a ação do mesmo sobre o sistema nervoso autônomo (SNA), com isso libera norepinefrina e secreta epinefrina, gerando aumento da frequência cardíaca, do metabolismo celular, e por consequência hiperglicemia, em um momento de estresse (MACHADO; HAERTEL, 2006).

Em um processo de entendimento do estresse descobriu-se que o ambiente onde o indivíduo está correlacionado, pode influenciar severamente em suas ações e no processo fisiológico dos seus sistemas, estando diretamente ligado ao estresse. Os universitários por sua vez, passam por situações de estresse diárias, que podem prejudicar sua saúde e qualidade de vida. Estes em meio a uma nova etapa de suas vidas, tem a necessidade de gerenciar uma vida social e pessoal, conciliando-as com as atividades acadêmicas e a preparação acerca de suas futuras carreiras (BORINE et al.,2015; BUBLITZ et al., 2016; LORENZO et al., 2013).

Cada indivíduo tende a enfrentar o estresse de forma singular, onde este pode gerar diversos efeitos fisiológicos que, a depender da resposta de enfrentamento do indivíduo, ou seja, dos seus esforços de adaptação aos estímulos gerados, as consequências podem ser graves. Todavia, o organismo pode se adaptar, muitas vezes por aparecimento de doenças onde há expressivo esforço e adaptação, como em gastrites e úlceras digestivas ocasionadas pelo estresse, além também de variações de pressão arterial, entre outros (ALMEIDA, 2010; SILVA et al., 2017).
O estresse em universitários gera muita carga mental e física a um indivíduo, principalmente na área da saúde, onde há o medo do enfrentamento da rotina, e ainda o temor em não conseguir exercer a profissão com qualidade pela falta de conhecimento prático ou teórico. Ademais, o fato de não ter uma forma de recreação atrapalha o cognitivo, trazendo características tardias e graves de depressão e ansiedade (HIRSCH et al., 2018).

\section{TRABALHOS CORRELATOS}

Diversos trabalhos relacionam-se em muitos aspectos com o tema abordado. Como pode ser observado, na Tabela 1, retrata que, de acordo com Ferraz e Gomes (2009) durante a fase de alarme, neutrófilos, monócitos, hemoglobina, hematócrito, cortisol e glicose tiveram suas quantidades aumentadas em relação aos padrões normais dessas células dentro da espécie utilizada na simulação, enquanto linfócitos apresentaram-se diminuídos.

\begin{tabular}{|c|c|c|c|}
\hline \multicolumn{4}{|c|}{ Indicadores sanguineos de repouso e estresse de acordo com diferentes autores } \\
\hline Indicadores/Autores & Dhabhar et al. (2012) & Maydych et al (2017) & Ferraz; Gomes, 2009 \\
\hline Epinefrina & & - & - \\
\hline Norepinefrina & †após 6 minutos & - & - \\
\hline Cortisol & $\begin{array}{c}\text { †após } 2 \text { minutos e } \\
\uparrow \text { mais ainda }\end{array}$ & †após 30 minutos & Aumento \\
\hline Linfócitos & $\begin{array}{l}\text { †durante } 6 \text { minutos } \\
\text { †após } 2 \mathrm{~h}\end{array}$ & $\uparrow \mathrm{CD8}$ & Diminuição \\
\hline Monócitos & $\ldots \ldots \ldots \ldots$ & $\begin{array}{l}\text { \do númerołda } \\
\text { funçăo }\end{array}$ & Aumento \\
\hline Neutrófilos & $\begin{array}{l}\text { †durante } 6 \text { minutos } \\
\text { †após } 2 \mathrm{~h}\end{array}$ & $\begin{array}{l}\text { †citocinas pró- } \\
\text { inflamatórias }\end{array}$ & Aumento \\
\hline Glicose & - & - & Aumento \\
\hline Hemoglobina & - & - & Aumento \\
\hline Hematócrito & - & - & Aumento \\
\hline
\end{tabular}

Dhabhar et al. (2012) e Maydych et al. (2017) também explanaram a respeito de indicadores sanguíneos de repouso e estresse em fase de alarme. Dhabhar et al. (2012), demonstrou em uma pesquisa com ratos de laboratório que a indução do estresse juntamente com os hormônios, como a epinefrina e a norepinefrina, são mais lentos para induzir o aumento das células do sistema imunológico quando comparado ao cortisol, uma vez que este pode estimular o aumento muito rapidamente mas, reprime as células imunitárias na mesma velocidade, algumas se mantêm estáveis ao passar do tempo, algumas diminuem, porém continuam exercendo sua função, mesmo que em menor quantidade. 
Já Maydych et al. (2017) fez sua pesquisa baseada também no estresse aplicado ao sistema imunológico, porém mais focado nas células como os linfócitos e o impacto crônico associado. Neste, participaram 39 alunos de uma universidade, os quais responderam um questionário com perguntas demográficas e assim, fizeram a coleta de sangue.

Como os autores supracitados, o resultado foi semelhante, onde o cortisol, um dos hormônios principais que causam o estresse aumentou significativamente e linfócitos do tipo CD8 aumentaram bem como células do tipo Natural Killers diminuíram com o passar do tempo. Também foi observado aumento o número de citocinas próinflamatórias acarretando maior números de células. Contudo, os monócitos diminuíram, uma vez que migram para os tecidos adjacentes, mesmo assim ficaram com sua função aumentada.

Junto as alterações hematológicas, podem ser observados aumentos e diminuições de diversos marcadores bioquímicos e fisiológicos nas três fases principais do estresse, como indicado por de Guest et al. (2013) (Tabela 2).

Tabela 2- Dados correlatos ao estresse sobre padrões bioquimicos e hematológicos

\begin{tabular}{ccc}
\hline \multicolumn{2}{c}{ Fases do estresse e caracteristicas de cada fase no sangue. } \\
\hline Fase de alarme/alerta & Fase de resistência & Fase de exaustão \\
\hline đde Noradrenalina e Adrenalina & $\uparrow$ Hormônio liberador de & corticotrofina \\
$\begin{array}{c}\uparrow \text { Ácidos graxos } \\
\text { Vatimentos cardiacos }\end{array}$ & $\uparrow$ Hormônio corticotrófico & $\uparrow$ Glicerol \\
Vasoconstrição & $\uparrow$ Cortisol & $\uparrow$ Leucócitos \\
Midríase & & \\
$\uparrow$ Glicose & & \\
\hline Fonte: Adaptado de GUEST et al. (2013).
\end{tabular}

Guest et al. (2013) mostram aumentos e diminuições de diversos hormônios e compostos, em relação aos padrões normais em humanos, onde seus metabolismos são diretamente prejudicados em cada fase de estresse. Neste estudo é observado que na fase de alerta há aumento de noradrenalina e adrenalina, glicose, por conseguinte, dos batimentos cardíacos do indivíduo, além de gerar também a vasoconstrição de vasos do indivíduo e o aparecimento de midríase no mesmo. Na fase de resistência, observou-se a ocorrência do aumento da liberação de hormônio liberador de corticotropina, de hormônio corticotrófico e de cortisol. Já na fase de exaustão, observou-se aumento de níveis de ácidos graxos, glicerol e de leucócitos.

Em um estudo mais específico referente ao estresse, realizado com 207 estudantes universitários dos cursos de Biomedicina, Fisioterapia, Enfermagem, Estética, Farmácia e Biologia, com base no Inventário de Sintomas de
"Stress" de LIPP (ISSL) em período de provas, demonstrou que 89 alunos (43\%) não apresentaram sintomas, ou seja, não estavam estressados, enquanto que 118 (57\%) se apresentaram em alguma fase de estresse (CHAVES et al., 2016) (Tabela 3).

Tabela 3 - Dados compilados quantitativos e porcentagens de alunos nas fases de alarme/alerta resistência, quase-exaustão e exaustão.

\begin{tabular}{cc}
\multicolumn{1}{c}{ Alunos identificados nas fases do estresse } \\
\hline Fases do estresse & Quantitativo e porcentagem \\
\hline Alarme/Alerta & 21 alunos $(18 \%)$ \\
Resistência & 71 alunos $(60 \%)$ \\
Quase-exaustão & 8 alunos $(6,7)$ \\
Exaustão & 18 alunos $(15,3)$ \\
Total & 108 alunos $(100 \%)$ \\
\hline Fonte: Adaptado de CHAVES et al. (2016).
\end{tabular}

Como observado na Tabela 3, a pesquisa de Chaves et al. (2016) indica que a maioria dos alunos universitários que estavam estressados $(60 \%)$ se encontravam na fase de resistência, onde há maiores sintomas de desgaste e cansaço, enquanto que uma minoria ( 8 alunos) se encontravam em fase de quase-exaustão, ou seja, onde há enfraquecimento do organismo e a perda de sua capacidade de adaptar-se a fatores estressantes bem como de resistir a eles, dando oportunidade ao aparecimento de doenças que se instalam na fase de exaustão. A quantidade de alunos em exaustão foi de 18 alunos, sendo esta quantidade inferior aos de alunos em fase de alarme ou alerta (21 alunos).

O estudo revela que o estresse está presente nos mais diversos meios sociais, distribuídos entre diferentes tipos de pessoas, mas que, em alguns casos, este acaba sobrepondo-se em determinados meios do que em outros. Variáveis individuais e culturais devem ser consideradas ao se avaliar o estresse entre diferentes organismos, já que, um dos principais fatores para se ter controle sobre o estresse é o processo de enfrentamento, que é inerente a cada indivíduo, dependendo dos recursos sociais e psicológicos de cada um (LIPP; FRARE; SANTOS, 2007).

O estresse pode gerar diferentes respostas em diferentes tipos de organismos, mas isto também dependerá do estímulo gerador, dessa forma, um modelo cognitivo proposto por Lazarus (1993) propõe que o organismo estressado deve fazer um reconhecimento de sua situação onde este será o ponto central na compreensão do enfrentamento do agente estressor. $\mathrm{O}$ reconhecimento deve ser feito observando-se as limitações, demandas e recursos do meio, além também dos objetivos e prioridades do indivíduo e suas crenças pessoais, assim, o valor 
das estratégias de enfrentamento dependerão da situação enfrentada (estímulo estressor), da situação estudada (saúde física, mental, trabalho por exemplo) e da personalidade do indivíduo (LAZARUS, 1993).

Chaves et al. (2016) também mostra (Tabela 3) a grande incidência de alunos estressados na graduação em período de provas dentro de um grupo de 118 alunos, onde, os alunos em fase de resistência estão em maior quantidade (71 alunos). A fase de resistência se inicia com a tentativa de adaptação do indivíduo para restabelecer sua homeostase (LIPP; MALAGRIS, 2011). A sensação de cansaço e desgaste aparece e os valores de marcadores hematológicos e imunológicos são alterados. Como visto na Tabela 2 , há o aumento nesta fase de produção de hormônio liberador de corticotrofina, hormônio corticotrófico e por consequência, cortisol, para o possível controle do estresse mas que já está gerando desregulação na homeostase corporal do organismo (FERRAZ; GOMES, 2009; FONSECA; GONÇALVES; ARAÚJO, 2015)

Apesar de Chaves et al. (2016) mostrar que apenas $15,3 \%$ dos alunos estão em fase de exaustão e que apenas $6,7 \%$ estão em fase de quaseexaustão, a tendência de que os 71 alunos (60\%) que estão em fase de resistência entrem em fase de quase-exaustão ou exaustão são grandes, uma vez, que, se tratando do meio acadêmico, o aluno é posto dentro de diferentes situações e diversos fatores estressantes que podem prejudicar sua saúde e qualidade de vida, já que, neste período, precisam gerenciar vida social e pessoal, conciliando-as com as necessidades acadêmicas, preocupações sociais, familiares e ainda suas futuras carreiras (BORINE et al., 2015; BUBLITZ et al., 2016; LORENZO et al., 2013).

Observa-se que, no estudo de Ferraz e Gomes (2009), retratou-se das diferentes concentrações de referenciais hematológicos e bioquímicos, onde os modelos de estudos (peixes) foram colocados sob diferentes interações sociais a fim de testar a influência nesses índices, e foi observado que na fase de alerta, onde estes sofreram estresse, os valores dos testes subiram significativamente, isso em comparação aos peixes controle, ou seja, que não foram expostos a diferentes interações sociais (Tabela 1 ).

Diante disso, fica claro que não só índices hematológicos podem mudar, mas os hormônios têm grande influência. Além disso, nos primeiros sinais de estresse, na fase de alarme/alerta o que mais se destaca é a adrenalina, que aumenta o fluxo sanguíneo e o cortisol, que pode inibir várias ações fisiológicas no corpo, até mesmo imunomodular negativamente os leucócitos na fase de exaustão (GUEST et al., 2013) (Tabela 2).

$\mathrm{Na}$ fase de exaustão, apresentada na Tabela 2, é possível observar que há aumento de leucócitos como os neutrófilos, provavelmente por serem os primeiros a adentrar a circulação sanguínea. Ainda, pode-se observar grande acúmulo de hemácias e hemoglobina, especula-se que é por conta da necessidade de maior oxigenação de tecidos durante o estresse. Cabe salientar a concomitante inibição de insulina, o que é um fato preocupante uma vez que a glicose se torna muito elevada na circulação sanguínea, podendo desencadear várias complicações para o indivíduo. E por fim, há também a super excitação das glândulas adrenais, as quais continuarão liberando cortisol (GUEST et al., 2013).

Na Tabela 1 os três autores investigam assuntos correlatos, onde avaliam o estado de repouso e o estresse em si, além dos valores dos aspectos hormonais e das células efetoras da imunidade. Pode-se observar que houve um aumento dos hormônios em questão, onde o cortisol tornou-se o protagonista, e fez com que células do sistema imunológico, como leucócitos, fossem imunomoduladas negativamente, ao ponto de voltarem ao seu nível basal, algumas ainda continuaram aumentando, com as do tipo CD8 citotóxicas e também células do tipo Natural Killers, que por sua vez agem atacando qualquer tipo de ameaça ao sistema imunológico, até as próprias células se preciso, porém o cortisol ainda conseguiu manter essas células com o tempo em diminuição, como observado em Maydych et al. (2017). Dhabhar et al. (2012) demonstraram diminuição das células como linfócitos e monócitos, enquanto os neutrófilos só aumentaram as quantificações, apenas voltavam ao estado basal com o aumento do cortisol. Em consonância, Ferraz e Gomes (2009), demonstraram o aumento no número de células efetoras do sistema imunológico, contudo, diminuição dos leucócitos.

Observando-se, através do presente estudo, é significativa a quantidade de fatores estressantes que podem vir a ocasionar prejuízos a um indivíduo além também de como esses fatores 
atuam dentro de um organismo, trazendo desequilíbrio homeostático, apresentando gatilhos para o surgimento de doenças autoimunes, diabetes, transtornos psicológicos e é até mesmo associado ao desenvolvimento de câncer.

O câncer é dito como uma doença multifatorial, possuindo estreita relação com qualidade de vida, fatores hormonais, hereditários, mutações genéticas, e ainda, fatores psicológicos, como o estresse (FARAGO et al, 2010; LEITE et al., 2016). Condições psicológicas estão intimamente relacionadas a liberação de cortisol e a qualidade de vida como dito anteriormente, e estando o cortisol relacionado ao estresse, por conseguinte, o próprio estresse possui íntima relação com o eixo hipotálamo-hipófise de um organismo, ademais, alguns estudos demonstram que, justamente por toda essa relação (hipotálamo-hipófise, cortisol e estresse), o estresse está relacionado ao aparecimento de neoplasias e diminuição da imunovigilância (FARAGO et al, 2010).

\section{CONSIDERAÇÕES FINAIS}

O estresse afeta o indivíduo como um todo, causando severos efeitos sobre seus parâmetros hematológicos, bioquímicos e imunológicos. Nesse sentido, alunos de graduação são os muito expostos sobre essas questões, devido a carga mental e física que precisam exercem durante o estudo e até mesmo, com a carga laboral.

Portanto percebe-se a necessidade de uma manutenção na qualidade de vida entre universitários, com acompanhamento psicológico e a avaliação dos parâmetros bioquímicos, hematológicos e imunológicos.

\section{REFERÊNCIAS BIBLIOGRÁFICAS}

ALMEIDA, O. M. M. S. A resposta neurofisiológica ao stress. In M. E. N. Lipp (Org.), Mecanismos neuropsicofisiológicos do stress: Teoria e aplicações clínicas. Casa do Psicólogo. 3a ed. São Paulo, p. 25-30, 2010.

BORINE, R. C. C.; WANDERLEY,, K. S.; BASSITT, D. P. Relação entre qualidade de vida e estresse em acadêmicos da área da saúde. Est. Inter. Psicol., Londrina, v.6, n.1, jun, .2015.

BUBLITZ, S.; GUIDO, L. A.; LOPES, L. F. D.; FREITAS, E. O. Association between nursing student's academic and sociodemographic characteristics and stress. Texto contexto - enferm., Florianópolis, v. 25, n.4, p.e2440015, dec., 2016. CHAVES, et al. Estresse em universitários: análise sanguínea e qualidade de vida. Revista Brasileira de Terapias Cognitivas. Rio de Janeiro, v. 12, n.1, jun. 2016.

DHABHAR, Firdaus S., et al. Stress-induced Redistribution Of Immune Cells - From Barracks To Boulevards To Battlefields: A Tale Of Three Hormones - Curt Richter Award Winner. Rev. Psychoneuroendocrinology. v. 37, n. 9, p. 1345-1368, set., 2012.

FARAGO, P. M. et al. My life breast cancer: report of emotional, stress. Revista de Enfermagem, UFPE, v. 4, n. 3, p. 10371042, 2010.

FERRAZ, Francielle Bonett. GOMES, Levy Carvalho. Social relationship as inducer of immunological and stress responses in matrinxã (Brycon amazonicus). Comparative Biochemistry and Physiology, Part A 153, p. 293-296, 2009.

FONSECA, N. C.; GONÇALVES, J. C.; ARAUJO, G. S. Influência do estresse sobre o sistema imunológico. Simpósio de TCC e Seminário de IC, 1/ 2015. São Paulo: ICESP, n 8, 2015.

GUEST, Francesca $L$, et al. Os efeitos do estresse na função do eixo hipotalâmico-pituitário-adrenal em indivíduos com esquizofrenia. Rev. Psiq. Clín., v. 40, n. 1, p. 20-7, 2013.

HIRSCH, Carolina Domingues, et al. Fatores Percebidos Pelos Acadêmicos De Enfermagem Como Desencadeadores Do Estresse No Ambiente Formativo. Texto Contexto Enferm, v. 27, n. 1, p. e0370014, 2018.

JURUENA, Mario F. CLEARE, Anthony J. PARIANTE, Carmine M. O eixo hipotálamo-pituitária-adrenal, a função dos receptores de glicocorticóides e sua importância na depressão. Rev. Bras. Psiquiatria, v. 26, n. 3, p.189-201, jun, 2004.

LAZARUS, R. S. From psychological stress to the emotions: A history of changing outlooks. Annual Review of Psychology, v. 44, p. 1-21, 1993.

LEITE, F. P. et al. Análise cienciométrica sobre a relação da vivência de eventos de vida produtores de estresse e desenvolvimento de câncer. Cinergis, v. 17, n. 3, 2016.

LIPP, M. E. N.; FRARE, A.; SANTOS, F. U. Efeitos de variáveis psicológicas na reatividade cardiovascular em momentos de stress emocional. Estudos de Psicologia, Campinas, v. 24, p. 161- 167, abril/junho, 2007.

LIPP, M.; MALAGRIS, L. Estresse. Aspectos históricos, teóricos e clínicos. In: RANGÉ, B. E. cols. Psicoterapias cognitivocomportamentais. Um diálogo com a psiquiatria. Porto Alegre: Artmed, 2011. 
MACHADO, Angelo B.M.; HAERTEL, Lúcia Machado. Neuroanatomia funcional. 3.ed. São Paulo: Atheneu, 2006.

MAITRA, A.; ABBAS, A. K. O Sistema endócrino. In: ROBBINS, S. L.; COTRAN, R. S.; KUMAR, V. Patologia estrutural e funcional. 9a ed., p. 1207 - 1282, Rio de Janeiro: Guanabara Koogan, 2016.

MAYDYCH, Viktoriya et al. Impact of chronic and acute academic stress on lymphocyte subsets and monocyte function.

Plos One, v. 12, n. 11, nov., 2017.

PAGLIARONE, Ana C. SFORCIN, José M. Estresse: revisão sobre seus efeitos no sistema imunológico. Rev. Biosaúde, Londrina, v. 11, n. 1, p. 57-90, jan./jun. 2009.

PRADO. C. E. P. Estresse ocupacional: causas e consequências. Rev. Bras. Med. Trab., v. 14, n. 3, p.285-289, 2016.

SILVA, et al. Lista de Sintomas de Stress Pré-competitivo Infanto-juvenil: Adaptação para Bailarinos. Revista Iberoamericana de Diagnóstico y Evaluación - e Avaliação Psicológica. RIDEP, v. 1, n. 43, p. 59-70, 2017.

STURION, A. L. T. Cortisol, glicose e triglicerídeo na avaliação do estresse neuroendócrino de cães com fratura tibial. Monografia (Mestrado em Medicina Veterinária) - Universidade Federal de Santa Maria. Santa Maria, p. 37, 2011. 